\title{
Contacto piel a piel inmediato al nacimiento y beneficios en el recién nacido sano en los primeros 30 minutos
}

\author{
Ceci Margoth Rios Mori, ${ }^{1, a}$ Mónica Patricia Lias Cuervo. ${ }^{2, a, b}$
}

Rios MCM, Lias CMP. Contacto piel a piel imediato al nacimiento y benefícios em el recién nacido sano em los primeiros 30 minutos. Cuid salud, jul-dic 2015; 2(2):177-186.

\section{RESUMEN}

El nacimiento es una etapa única para el neonato y su madre; quien experimenta un cambio brusco al nacimiento, que podría minimizarse colocándolo sobre el abdomen-pecho materno al momento de nacer y beneficiar su adaptación fisiológica y psicológica al ambiente externo. Objetivo: determinar los beneficios del contacto piel a piel en el recién nacido $(\mathrm{RN})$ sano durante los primeros 30 minutos inmediato al nacimiento. Metodología: estudio de diseño cuasiexperimental, con una muestra de $50 \mathrm{RN}$ sanos por grupo, aplicando los criterios de inclusión y exclusión, previo consentimiento informado de la madre. El instrumento fue una lista de observación que midió la adaptación fisiológica y psicológica del neonato, salvaguardando los principios de la ética en investigación. Para el análisis se utilizó la prueba estadística de Mann Whitney. Resultados: el $96 \%$ de RN sanos logró succión inmediata a partir de los 10 minutos colocados sobre el abdomen-pecho materno, además de estabilizar sus funciones vitales de respiración y temperatura, con diferencia entre grupos $(p=0,00)$. Conclusiones: el contacto piel a piel mejoró categóricamente la adaptación fisiológica y psicológica del $\mathrm{RN}$ en el grupo experimental. Es necesario sensibilizar y capacitar al profesional de salud para cumplir la norma y contribuir con la sobrevida del neonato.

Palabras clave: contacto piel a piel, recién nacido sano, desarrollo psicológico, enfermería.
Rios MCM, Lias CMP. Skin-to-skin contact immediate to the birth and benefits in the healthy newborn in the first 30 minutes. Cuid salud, jul-dic 2015; 2(2):177-186.

\section{ABSTRACT}

Birth is a unique stage for the newborn and his mother who experiences a sudden change to the birth, which could be minimized by placing it on the maternal abdomen-breast at birth and benefit from its physiological and psychological adaptation to the external environment. Objective: To determine the benefits of skin-to-skin contact in the healthy newborn (NB) during the first 30 minutes immediate to birth. Methodology: A quasi-experimental design study with a sample of 50 healthy NB per group, applying the inclusion and exclusion criteria, prior informed consent of the mother. The instrument was an observation list that measured the physiological and psychological adaptation of the neonate safeguarding the principles of research ethics. Statistical analysis of Mann Whitney was used for the analysis. Results: $96 \%$ of healthy NB achieved immediate suckling after 10 minutes when they were placed on the maternal chest-abdomen, in addition to stabilizing their vital functions of breathing and temperature, with a difference between groups $(\mathrm{p}=0.00)$. Conclusions: The skin-to-skin contact improved categorically the physiological and psychological adaptation of the NB in the experimental group. It is necessary to sensitize and to train health professional to meet the standard and contribute to the survival of the neonate.

Keywords: Skin-to-skin contact, healthy newborn, psychological development, nursing.

\footnotetext{
1 Hospital Regional de Loreto Felipe Santiago Arriola Iglesias.

2 Hospital Nacional de la PNP Luis N. Sáenz.

a Licenciada Enfermera.

b Especialista en Neonatología.
} 


\section{INTRODUCCIÓN}

La UNICEF-India, en concordancia con Gangal et al., ${ }^{1}$ refiere que el contacto piel a piel incrementa la alimentación de pecho de manera amigable y satisfactoria, promoviendo una respuesta afectiva entre madre-neonato que los une emocional y fisiológicamente. Tal proceso debe realizarse inmediatamente después del nacimiento con el neonato sano, colocándolo en el pecho y abdomen desnudo de su madre; ${ }^{1}$ acción que reduce el $22 \%$ de todas las muertes entre los neonatos menores de un mes en los países en vías de desarrollo. $^{2}$

Klaus et al., citado por Pinto, ${ }^{3}$ demostraron en sus estudio que los neonatos del grupo experimental que permanecieron encima del pecho materno durante los primeros 45 minutos de vida mantuvieron una buena lactancia natural exclusiva, con mayor ganancia de peso al sexto mes y menor número de procesos infecciosos. Resultados que fueron corroborados en diversos estudios alrededor de todo el mundo. ${ }^{4}$

Al no existir contraindicación médica durante el nacimiento es fundamental que la madre permanezca al menos los primeros 30 minutos en intimo contacto piel a piel con su neonato desnudo entre su abdomen-pecho, acto que permitirá que el $\mathrm{RN}$, a través de su olfato y movimientos reptantes, se aproxime al pecho (pezón-areola) y comience a succionar, estimulando así la producción de las hormonas prolactina y oxitócina, tan beneficiosas para el apego, la lactancia y la prevención de hemorragias uterinas después del parto. ${ }^{1,5,6}$

La transición de la circulación fetal a la respiración autónoma es el cambio fisiológico más profundo que se le exige al neonato al nacer; esta desconexión significa perder el soporte metabólico completo, en especial del suministro de oxígeno y de la eliminación de dióxido de carbono; dichos cambios producen estrés durante el alumbramiento y alteran los patrones de intercambio gaseoso en el equilibrio acido-base de la sangre y en la actividad cardiovascular del neonato. ${ }^{7}$ Evidenciándose que la adaptación del RN a la vida extrauterina es un complejo proceso fisiológico.

Existe mucha información de contacto piel a piel en RN de bajo peso al nacer denominados madre canguro. Una revisión sistemática de la Chochrane Library ${ }^{8}$ concluye que este método reduce la morbi-mortalidad del $\mathrm{RN}$ de bajo peso al nacer, sugiriéndose como una alternativa frente al cuidado convencional en ambientes con recursos limitados. Mientras tanto, es escaza la información de esta práctica en el RN sano, a pesar de formar parte de los protocolos de atención en los hospitales amigos del niño, promovidos por la UNICEF desde la década del 90, especialmente en los países en vías de desarrollo por la alta morbimortalidad infantil en los primeros meses de vida. ${ }^{1,6}$ Entre los beneficios del contacto piel a piel destacan la mejora de la interacción que favorece el vínculo, apego, reduce el llanto al nacer, estabiliza las funciones fisiológicas y psicológicas, ${ }^{1,59}$ mejorando ostensiblemente su condición de vida y sobrevivencia.

En la actualidad, el Ministerio de Salud (MINSA), en la Norma Técnica sobre la Atención Integral de la Salud Neonatal establece un conjunto de intervenciones articuladas para la disminución de la mortalidad neonatal, haciendo énfasis en la lactancia materna inmediata dentro de la primera hora del parto con los neonatos sanos (contacto piel a piel), reconociendo su alta vulnerabilidad y la importancia de una buena adaptación extrauterina. ${ }^{10}$ En ese sentido, el contacto piel a piel constituye una fase ineludible y de impacto en la vida y desarrollo del RN sano, debiendo la madre ser preparada y sensibilizada desde la gestación, promoviendo lo beneficios de esta práctica y el éxito en la lactancia natural exclusiva, proceso en el cual puede incluirse a la pareja para brindar todo su apoyo durante el nacimiento del neonato y ser parte de este instinto natural.

Empero, a nivel mundial pocos son los países que lo aplican. La OPS ${ }^{6}$ citando a Belizan et al., reporta que los médicos no implementan esta práctica por falta de acceso a la información científica o por la incapacidad de entender la literatura científica, también aluden falta de tiempo o de recursos físicos, además de actitudes de resistencia al cambio, falta de guías o existencia de políticas conflictivas. Situación semejante puede estar ocurriendo en el país, dónde pocos son los hospitales que desarrollan esta actividad como parte de la rutina. Hecho que motiva la aplicación del método en este estudio y el escaso recurso humano capacitado para ejecutarlo, con el fin de sensibilizar a los profesionales que laboran en neonatología y promover su inserción en la cotidianidad de los servicios del primer nivel de atención de salud.

Por las consideraciones expuestas, el objetivo del estudio fue determinar los beneficios del contacto piel a piel en el recién nacido sano 
durante los primeros 30 minutos inmediato al nacimiento, en el servicio de neonatología del Hospital Aurelio Díaz Ufano y Peral-EsSalud, en Lima-Perú.

\section{Contacto piel a piel}

El contacto piel a piel es la acción donde dos cuerpos se tocan; se roza la piel del $\mathrm{RN}$ con la de la madre. El primer contacto es ideal para iniciar la primera lactancia de forma natural y comienza con el arrastre del neonato hacia al pecho y termina con el agarre del pezón (areola), iniciando de esta manera la primera mamada.,

Es un método que favorece el vínculo materno-neonato $\mathrm{y}$, sobre todo, la condición fisiológica y psicológica del neonato, siendo también la madre favorecida mediante los movimientos reptantes del $\mathrm{RN}$ sobre su vientre durante este contacto, ayudándole a desprender de forma natural la placenta, ${ }^{11}$ disminuyendo la pérdida de sangre post-parto., ${ }^{5,6,9}$

El contacto piel a piel madre-neonato reduce el llanto al nacer, mejora la interacción afectiva entre la madre y su RN, manteniéndolo caliente y ayudando a la mujer a dar de lactar con éxito; ${ }^{11}$ entre otros beneficios que mejoran el estado de salud de la diada.

Según Gangal et al., ${ }^{1}$ el neonato al ser colocado en contacto piel a piel sobre la madre, ella le pasará los microorganismos de su cuerpo para que mantenga su protección y disminuya el riesgo de contraer alguna enfermedad neonatal.

El contacto piel a piel consiste en colocar al neonato desnudo después del nacimiento en posición decúbito ventral sobre el abdomen-pecho desnudo de la madre, cubriendo su espalda con una manta (templada) para que ambos sientan el primer contacto físico y compartan la experiencia afectiva, ${ }^{10}$ posiblemente inducida por el alto componente de oxitócina en la diada, además de catecolaminas en el RN. Alba et al., ${ }^{9}$ en su estudio de revisión afirman que estas hormonas serían las responsables de la intensa sensación amorosa que experimenta el RN durante las primeras horas de vida y que quedarán grabadas en varias áreas de su cerebro; sensación que podría también estar experimentando la madre; haciendo de este evento un acto instintivo, único y perdurable.

\section{Beneficios en el recién nacido sano}

En diversas investigaciones el contacto piel a piel inmediato (CPPI) brinda múltiples beneficios para la diada madre-neonato, destacando que no solo logra una mejor transición de la vida intrauterina a la extrauterina, sino que favorece los cambios inmediatos que ocurren en el neonato durante este período adaptativo. ${ }^{5,9,12}$

En el aspecto psicológico le ayuda a mantener la estabilidad de vida emocional saludable y amigable con la madre, unión que no debe ser interrumpida porque se estaría rompiendo un lazo afectivo y favorable, conociéndose mutuamente y estableciendo diálogos subliminales. ${ }^{9,12}$

Esta comunicación inmediata entre la diada promueve la comunicación verbal, gestual y no verbal, en la que intervienen el tacto, el olfato, la visión e incluso el gusto., ${ }^{5,9,12}$ Mediante el tocamiento de las manos sentirán una atracción y unión afectiva y las palabras de la madre estimularan la sensación auditiva del neonato, quien reconocerá su voz de forma inmediata, sintiéndose más seguro y amado; calmando así su llanto y permaneciendo tranquilo. De esta manera el neonato podrá mantener un buen sueño y no se sentirá estresado por el cambio que tuvo al pasar a la vida extrauterina., 5

En el aspecto fisiológico, el neonato recibe beneficios en la mejor regulación de la temperatura, respiración, frecuencia cardiaca y saturación de oxígeno. Al nacer ocurre un cambio brusco en su temperatura corporal que es $<36,5^{\circ} \mathrm{C}$ siendo incapaz aun de poner en funcionamiento su sistema hipotalámico; proceso que es muy bien compensando con el calor corporal de la madre por el mecanismo de conducción térmica, al ser colocado en contacto piel a piel sobre su abdomenpecho, hecho que se produce gracias la respuesta térmica inmediata de la madre ${ }^{13}$ y la intervención de varias hormonas, entre ellas la oxitócina y las endorfinas. $^{11}$

\section{MATERIAL Y MÉTODOS}

\section{Diseño de estudio y área de ejecución}

Estudio de enfoque cuantitativo, método experimental y diseño cuasiexperimental, realizado en el Centro Obstétrico del Hospital Aurelio Díaz Ufano y Peral, perteneciente a la Red Almenara del Seguro Social de Salud (EsSalud) en LimaPerú, ubicado en el Jr. Rio Mar S/N, Urb. Los Pinos, distrito de San Juan de Lurigancho. El alcance del hospital es netamente urbano, siendo clasificado como una institución de Nivel I de 
atención y ante cualquier situación de complejidad, la madre y el recién nacido son transferidos al Hospital Nacional Guillermo Almenara-EsSalud. El Centro Obstétrico brinda atención las 24 horas del día a las gestantes que deriven en parto eutócico, quienes son usuarias de EsSalud y pertenecen a la clase socioeconómica media y baja.

El Centro Obstétrico o sala de parto se ubica en la planta baja, cuenta con un equipo de profesionales capacitados: médicos ginecólogos, obstétrices y enfermeras especialistas en neonatología, además de médicos neonatólogos y pedíatras; correspondiendo a las enfermeras recibir al RN sano y a quien proveen de cuidados inmediatos, en trabajo colaborativo con el médico neonatólogo.

\section{Población y muestra}

La población fue conformada por todos los RN sanos de parto eutócico. Según datos del Registro Estadístico del Hospital existían de 150 a más nacimientos por mes hasta enero del 2011, incluyendo las gestaciones a término de 37-42 semanas. Sobre este dato fue estimado que habría aproximadamente 450 nacimientos por trimestre; sin embargo, tratándose de un diseño cuasiexperimental se optó por estudiar en cada grupo a $50 \mathrm{RN}$ sanos, conforme a los criterios de inclusión: consentimiento informado de la madre o gestante firmado, RN sano de parto eutócico, entre 37-42 semanas y, el criterio de exclusión fue: RN sano con bajo peso al nacer o RN con presencia de alguna patología.

\section{Instrumentos y variables}

El instrumento utilizado para recolectar los datos fue una lista de chequeo que contenía las variables en estudio: contacto piel a piel y beneficios; siendo sometido a validez mediante juicio de expertos conformado por 10 especialistas entre médicos y enfermeras además de un profesional estadístico; los resultados de este proceso fueron analizados con la prueba Binomial $(\mathrm{p}<0,05)$. Posteriormente, el instrumento se sometió a confiabilidad mediante prueba piloto en una muestra de $15 \mathrm{RN}$ sanos, cuyos datos fueron analizados mediante la prueba Kuder y Richardson $(\mathrm{p}=0,99)$ y la prueba de Tukey para las varianzas entre "tiempo cero" (iniciado una vez colocado el RN en el abdomen-pecho materno) 10, 20 y 30 minutos después del nacimiento. Finalmente, el instrumento quedo conformado de tres partes: Presentación, Datos generales y observaciones a realizar de la variable dependiente en estudio (beneficios), con 14 ítems con respuestas SI-NO, considerando valores de uno (1) para la respuesta positiva y cero (0) para respuesta negativa.

La variable "contacto piel a piel" fue definido como la unión del RN sano con el abdomen-pecho de la madre inmediato al nacimiento, estableciendo un vínculo entre ambos que permite al RN arrastrarse hacia arriba al reconocer el olor de la madre con su sentido del olfato altamente desarrollado, el cual le ayudará a llegar al pezón por medio del tacto y de movimientos de arrastre, dando inicio a la succión y primera mamada.

En la variable "beneficios" fueron evaluados los cambios ocurridos en la adaptación psicológica y fisiológica del RN sano al entrar en contacto con el abdomen-pecho materno considerando: menor tiempo de llanto, vigorosidad, buena coloración de la piel, temperatura apropiada, frecuencia respiratoria y cardiaca estables, óptima saturación de oxígeno, movimientos activos de miembros superiores e inferiores, gestos/muecas, movimientos de arrastre, reflejo de succión, sosiego y tranquilidad del neonato, seguido de sueño/descanso de la diada.

Indicadores de medición fisiológica: temperatura, frecuencia cardiaca, frecuencia respiratoria, saturación de oxígeno, movimientos de miembros superiores, movimientos de miembros inferiores, gestos/muecas, color rosadocianótico de piel, reflejo de succión y movimiento de arrastre.

Algunos ítems fueron observados en el RN al estar sobre el abdomen-pecho de la madre y luego en el cunero.

\section{Procedimiento de recolección}

Para la recolección de datos se obtuvo permiso del Director de la Red Almenara y luego, del Director del Hospital Aurelio Díaz Ufano de San Juan de Lurigancho y de la Jefa de Enfermeras del área del estudio, recolectando los datos entre abril y noviembre del 2011, previa coordinación con el personal profesional del Centro Obstétrico y de Neonatología.

Se utilizó como técnica la revisión documentaria y la observación participante, además de diversos materiales: termómetros rectales, termómetros plegables frontales, estetoscopio, reloj, cable de oximetría, pulsoxímetro neonatal, guantes quirúrgico $\mathrm{y}$ guantes desechables. 
Las historias clínicas fueron revisadas para obtener información sobre las gestantes y poder contactar con la futura madre y luego con el RN sano, siendo importante que la gestante firme la hoja de consentimiento informado y autorice la presencia de la investigadora en el proceso de parto.

En el mes de abril, la investigadora participó como observadora en los servicios de Neonatología y Centro Obstétrico junto a los profesionales de salud para conocer la cotidianeidad de los servicios, sensibilizando a los profesionales sobre la importancia de educar a la gestante en el método contacto piel a piel y su participación, considerado los materiales a usar en el estudio. En este periodo también se capacitaron a las enfermeras neonatólogas para recibir al RN sano que conformarían el grupo control.

Luego, en mayo se inició la recolección de datos con las gestantes internadas en el Centro Obstétrico, quienes fueron elegidas mediante muestreo no probabilístico, de tipo convencional. Previamente las gestantes recibieron explicación correspondiente sobre el estudio, disipando sus inquietudes; luego firmaron la Hoja de Consentimiento Informado (HCI). Para dividir a las gestantes entre el grupo experimental y control, todos los casos atendidos por la investigadora conformaron el grupo experimental y aquellos que se presentaban simultáneamente conformaron el grupo control, siendo evaluados por la enfermera capacitada en el marco de la rutina del servicio. A continuación se describe las actividades realizadas en cada grupo:

En el grupo experimental, la gestante era captada en el Centro Obstétrico, explicándole sobre la importancia del método contacto piel a piel, los beneficios para el RN sano, el tiempo que llevaría tomar las medidas del estudio y los procedimientos a ejecutar una vez nacido el neonato durante el parto. Ya en sala de parto, se permanencia junto a la madre antes, durante y después del parto; iniciado la fase de alumbramiento se esperaba la salida completa del neonato, a fin de recibirlo y recolectar los datos del estudio (según indicadores) en los cuatro momentos: al nacimiento, a los 10,20 y 30 minutos.

Inmediatamente al nacimiento el RN sano fue colocado en el abdomen-pecho desnudo de la madre en decúbito ventral, cubriendo su dorso con una manta o pañal de tela tibio para evitar la pérdida de calor, realizando aspiración boca-nariz si fuera necesario- y observando su estabilidad cardiorespiratoria. La madre debía sostener al neonato con sus brazos motivándolo a mantener un diálogo afectivo con el $\mathrm{RN}$; durante estos momentos se procedió con las medidas del estudio, observando su comportamiento y llegada al pezón para lactar. El corte umbilical fue realizado después de 3-4 minutos permitiendo el pase de mayor cantidad de sangre hacia el neonato, procedimiento que se ejecutaba sin interrumpir el contacto piel a piel. Pasado los 30 minutos, se continúo con los cuidados del RN en el cunero o servocuna: secado y limpieza, aplicación de gotas oftálmicas, anticoagulante IM, control de temperatura, medidas antropométricas y evaluación céfalo-caudal.

Terminado el parto la diada era conducida a sala de alojamiento conjunto permaneciendo con ellos durante 10-15 minutos más, haciéndole entrega a la madre de la copia de la HCI sellado con las huellas de los pies del RN; durante este tiempo se observaba el estado psicológico y fisiológico del RN mediante: facies, sosiego, lactancia o sueño; reforzado la necesidad de continuar con la lactancia materna exclusiva a libre demanda $y$, si estaban los familiares, se les orientaba brindar apoyo a la diada, haciéndose entrega de un tríptico con las enseñanzas básicas.

En el grupo control, una vez expulsados el RN sano del canal vaginal se realizaba la aspiración boca-nariz -si fuera necesarioevaluando su estabilidad cardiorespiratoria y luego se procedía con el corte umbilical, luego el RN era recibido por la enfermera neonatologa en una manta o pañal de tela tibio y trasladado al cunero o servocuna para proceder con los cuidados inmediatos: secado y limpieza, evaluación física, gotas oftálmicas, anticoagulante IM, control de temperatura, medidas antropométricas y al final, presentación del RN a su madre.

Posteriormente, el RN era conducido al servicio de Neonatología, donde se terminaban los cuidados; procediendo a vestirlo y colocarlo en el cunero o servocuna, cubierto por una colchita para mantener el calor. Pasado aproximadamente 1-2 horas el RN era conducido junto a la madre al Centro Obstétrico para alojamiento conjunto y dar inicio a la primera lactancia. En todo este proceso la enfermera del servicio cumplió con las medidas establecidas en el estudio, iguales a las del grupo experimental. 
Análisis de datos y aspectos éticos

Los datos fueron tabulados manualmente en Programa Excell 2004 mediante el uso de códigos y luego, transportados el Programa SPSS versión 15.0 para analizarse con la estadística descriptiva las univariables y comparar las medias de los grupos con la prueba Mann Whitney, considerando un $p<0,05$ de significancia.

El proyecto fue evaluado por el comité de investigación del Hospital Nacional Guillermo Almenara Irigoyen, otorgando un pareceré favorable mediante Carta $\mathrm{N}^{\circ}$ 828-OCID-G-RAAEsSalud-11. Durante el estudio se respetaron los principios de autonomía, beneficencia, no maleficencia y justicia propios de investigación con seres humanos, destacando que los datos obtenidos solo fueron utilizados para los fines aprobados.

\section{RESULTADOS}

Tabla 1. Contacto piel a piel según dimensiones en el grupo experimental. Hospital Nacional Díaz Ufano, mayo-dic 2011.

\begin{tabular}{|c|c|c|c|c|c|c|c|c|c|c|}
\hline \multirow[b]{2}{*}{ Dimensiones } & \multicolumn{2}{|c|}{ Nacimiento } & \multicolumn{2}{|c|}{$10^{\prime}$} & \multicolumn{2}{|c|}{20} & \multicolumn{2}{|c|}{$30^{\prime}$} & \multicolumn{2}{|c|}{ Total } \\
\hline & $\mathrm{Si}$ & No & $\mathrm{Si}$ & No & $\mathrm{Si}$ & No & $\mathrm{Si}$ & No & $\mathrm{N}$ & $\%$ \\
\hline \multicolumn{11}{|l|}{ Pecho } \\
\hline Succión inmediata & & 50 & 48 & 2 & 50 & & 50 & & 50 & 100 \\
\hline \multicolumn{11}{|l|}{ Abdomen } \\
\hline Arrastre por olor & & 50 & 37 & 13 & 50 & & 50 & & 50 & 100 \\
\hline
\end{tabular}

En la tabla 1 se identifica que casi todos los RN sanos del grupo experimental realizaron la succión del pezón a los 10 minutos $(96 \%)$ y, a los 20 minutos esta acción fue realizada por el $100 \%$ de RN. Inicialmente, los RN comienzan a realizar movimientos de arrastre y búsqueda hacia el pezón de la madre, conducidos por el olor que ella emana y que los bebes saben reconocerlo.

Gráfico 1. Comparación entre grupos de estudio según adaptación fisiológica. Hospital Nacional Díaz Ufano, mayo-dic 2011.

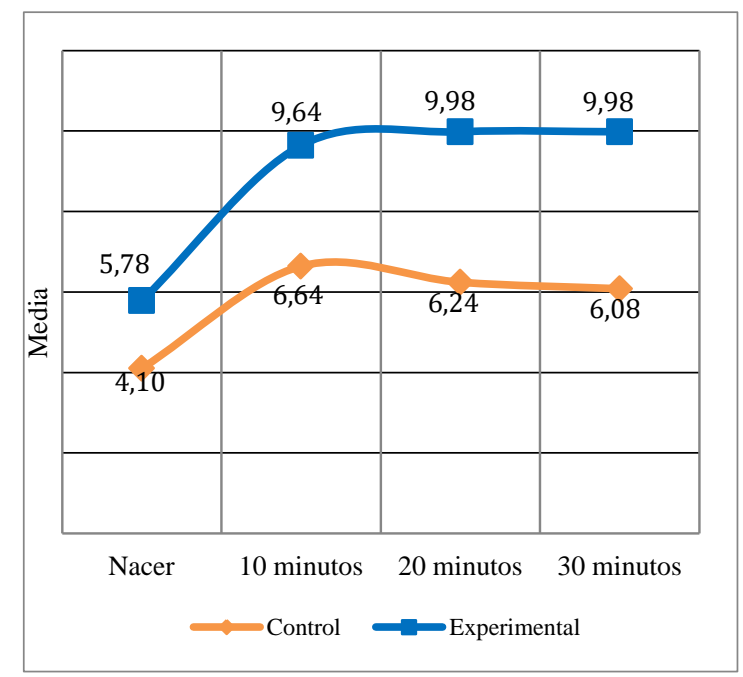

${ }^{*} \mathrm{p}<0,05$ existe diferencias significativas Prueba de Mann Whitney
El gráfico 1, se observa que al minuto de nacer el proceso de adaptación fisiológica fue mejor en el grupo experimental $(5,78)$ versus el grupo control $(4,1)$; a los 10 minutos esta mejora se amplía en el grupo experimental $(9,64)$ estabilizándose hacia los 20 y 30 minutos $(9,98)$ en comparación con el grupo control, que tiene una tendencia a caer $(6,24$ y 6,08$)$, con diferencia significativa entre grupos $(\mathrm{p}<0,05)$, en los cuatro momentos evaluados.

Gráfico 2. Comparación entre grupos de estudio según adaptación psicológica. Hospital Nacional Díaz Ufano, mayo-dic 2011.

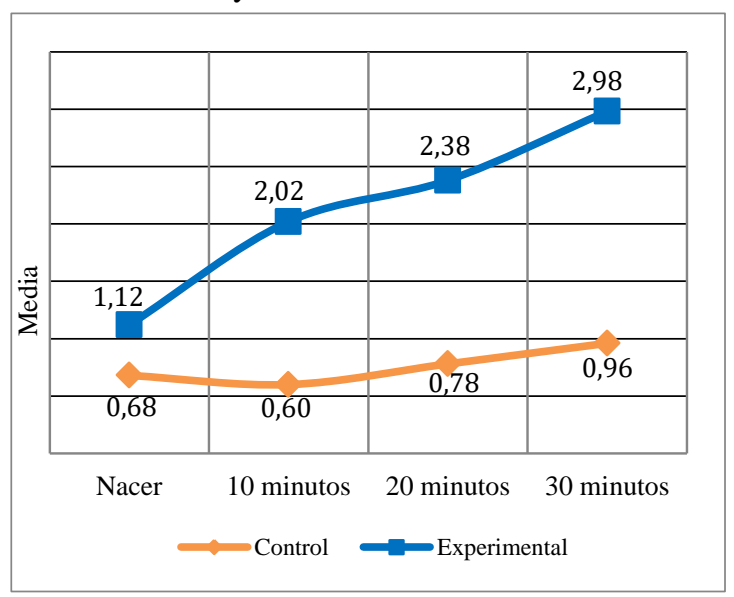

${ }^{*} \mathrm{p}<0,05$ existe diferencias significativas Prueba de Mann Whitney 
En el gráfico 2, se observa que al nacer la adaptación psicológica del RN fue mejor en el grupo experimental $(1,12)$, respuesta que continua en línea ascendente con el correr del tiempo $(2,02-$ 2,38-2,98). Adaptación que fue más lenta en el grupo control, con tendencia a desmejorar en los primeros 10 minutos, abrumados por el llanto y desasosiego; luego, existe una leve calma en los RN hasta los 30 minutos, pero continua el llanto y desasosiego; identificándose diferencia significativo entre grupos $(\mathrm{p}<0,05)$ en los cuatro momentos evaluados.

\section{DISCUSIÓN}

Los resultados del estudio muestran los beneficios inmediatos que el RN sano logra al entrar en contacto con el abdomen-pecho desnudo de la madre, a la par que la enfermera realiza los cuidados inmediatos impostergables en el RN, cubriéndolo siempre con una manta tibia para evitar la pérdida de calor corporal, al mismo tiempo que el RN reconoce el olor de la madre y comienza sus movimientos de arrastre, tanto que los beneficios de adaptación fisiológica y psicológica se observan desde los primeros minutos (gráfico 1 y 2), los cuales se consolidan con el pasar del tiempo.

En la adaptación fisiológica resaltan la temperatura ( $>35,5^{\circ}$ centígrados) y la saturación de oxigeno $(>90 \%)$ a los pocos minutos en la mayoría de los neonatos comparándolos con el grupo control. Respecto a la adaptación psicológica en los cuatros indicadores evaluados los RN sanos del grupo experimental muestran beneficios casi inmediatos, dejando el llanto prontamente con el correr del minuto, luego quedan calmados y sosegados totalmente a los 20 minutos, quedando adormilados a los 30 minutos, hecho que no ocurre en los RN del grupo control, que permanecen inquietos y llorando (gráfico 3).

Rivara et al. ${ }^{14}$ refieren que el contacto piel a piel del RN con su madre lo ayuda en la estabilización de la temperatura, la frecuencia cardiaca y respiratoria. Información que se sustenta en la revisión sistemática realizada por Moore et al. ${ }^{4}$ quienes fundamentados en 34 estudios randomizados y 2177 madres y sus RN, concluyen que el contacto piel a piel temprano favorece la lactancia materna y la estabilidad cardiorespiratoria, además de disminuir el llanto del lactante, sin conocerse efectos negativos a corto y largo plazo. Comprobándose además que existe una mejor interacción madre-neonato, con posibilidad que la lactancia se realice entre el primer y cuarto mes, con tendencia a extenderse por más tiempo. Datos que se corroboran en el estudio, excepto por la función respiratoria que tomó un poco más de tiempo para estabilizarse.

Ante la vulnerabilidad del RN, el contacto piel a piel busca protegerlo, cuidarlo y sobre todo, ayudarlo a sobrevivir. Así como los neonatos prematuros están en riesgo de morir por su inmadurez fisiológica, los neonatos a término también están en riesgo de adquirir cualquier enfermedad dentro y fuera del hospital; Lawm et al. ${ }^{15}$ refieren que la mortalidad es muy alta durante las primeras 24 horas después del nacimiento (25$40 \%$ ); atribuyéndose como causas de mortalidad neonatal a las infecciones en $36 \%$ (sepsis, neumonía, tétanos y diarrea) y al parto prematuro en $27 \%$.

Gangal et al. ${ }^{1}$ mencionan que el $\mathrm{RN}$ es vulnerable a cualquier enfermedad y quien mejor que la madre para ayudarle mediante el contacto piel a piel a estabilizar sus funciones biológicas y crear resistencia a los riesgos intrahospitalarios consumiendo las sustancias inmunes que contiene el calostro de la leche materna, además de beneficiarse de la flora bacteriana de la madre. Ambos le ofrecen protección contra las infecciones.

En Indonesia, el Hospital Dustira implementó el inicio temprano de la lactancia materna durante la primera hora del nacimiento. Un estudio que evalúa este procedimiento en la práctica identificó que el $91,8 \%$ de los RN sanos de la población estudiada realizó esta acción con éxito, principalmente entre los 30-44 minutos $(52,7 \%)$ y el 26,7\% entre los 15-29 minutos, resaltando que el $89,1 \%$ de los neonatos había nacido entre las 37-42 semanas de gestación. ${ }^{16}$ En el estudio, el $100 \%$ de los RN sanos había logrado amamantarse a los 30 minutos, destacando que el $74 \%$ de neonatos del grupo experimental tenía entre 37-39 semanas de gestación.

Según Ayukarningsih y Dwimanda, ${ }^{16}$ citando a Orun et al., durante los primeros 30 minutos los $\mathrm{RN}$ sanos experimentan una alerta silenciosa sin moverse y fortuitamente abren los ojos; en esta etapa experimentan su adaptación desde adentro hacia afuera del útero, utilizando entradas sensitivas y salidas motoras para arrastrarse, lo que generalmente ocurre entre los 27-71 minutos. Los RN del estudio lograron movimientos de arrastre aproximadamente a los 10 minutos $(90,4 \%)$, 
posiblemente en respuesta al estímulo táctil y auditivo que realizaba la madre al abrazarlo y hablarle suavemente mientras reposaba sobre su abdomen-pecho. La literatura reporta que existe una cierta relación de amor/apego en esta fase, gracias a las hormonas que se producen durante este periodo, principalmente la oxitócina endógena a nivel cerebral, que se sobrecargan tanto en la madre como en el neonato, produciendo sensaciones de confianza, bienestar, vivencia morosa y memoria social. ${ }^{9}$

Alba et al., ${ }^{9}$ refieren que el apego y la lactancia natural son importantes para ayudar a mantener un buen vínculo madre-neonato desde el inicio. Las madres en general se sienten emocionadas al formar parte de este proceso. ${ }^{17}$ Según Alba et al., ${ }^{9}$ las mujeres de todo el mundo se sienten emocionadas de contar sus historias del parto con otras mujeres en las semanas siguientes, resaltando frecuentemente sentimientos de vivencia amorosa.

La OMS/UNICEF incentivan el contacto piel a piel en los recién nacidos sanos. ${ }^{1,18}$ Sin embargo, muchos profesionales en el contexto nacional no lo aplican en sus centros laborales, a pesar de la existencia de la norma técnica, ${ }^{10}$ en la cual se ofrecen las pautas específicas para el RN sano, una vez comprobado su vigorosidad. Un estudio realizado en un hospital nacional materno infantil de Lima mostró que en la atención integral al RN no es prioridad cumplir con el contacto piel a piel, ya que el $96 \%$ de los neonatos permaneció sobre el pecho-abdomen desnudo de su madre menos de 15 minutos, ${ }^{17}$ a pesar de identificarse como uno de los hospitales amigo del niño en el país.

Realidad que muestra la necesidad de sensibilizar y capacitar al recurso profesional para cumplir con las metas de la atención integral al $\mathrm{RN}$, aunados a políticas claras y definidas por parte de los gestores en salud y aún más, haciendo seguimiento de su implementación y práctica en la función diaria. Considerando que existe suficiente evidencia científica de los beneficios que trae el método de contacto piel a piel a la diada madreneonato, ${ }^{5}$ pero especialmente al neonato por la alta mortalidad infantil que aún prevalece en el país, sobre todo cuando se evalúan a las poblaciones por quintiles.

Correspondencia:

Ceci Margoth Rios Mori

Correo electrónico: nursececita@gmail.com
El estudio muestra que una buena preparación de la madre, en el periodo inmediato al trabajo de parto, logra sensibilización de su parte y, por lo tanto, una estrecha colaboración inmediata al nacimiento, obteniéndose un vínculo madre-neonato efectivo durante el contacto piel a piel, produciendo elevada respuesta en el RN sano en todas los indicadores medidos (fisiológicas y psicológicas); siendo los resultados consistentes con previos estudios, pero a la vez, muestra mayores logros en menos tiempo.

Compete a las autoridades sanitarias y a los propios profesionales que trabajan en neonatología y sala de parto participar convincentemente de este proceso altamente saludable, que reduce la morbimortalidad infantil, especialmente en los países de bajos recursos económicos como el Perú $\mathrm{y}$, sobre todo, en las regiones de mayor riesgo como la Sierra y la Selva.

Entre las limitaciones que se reconocen en el estudio destacan su tamaño muestral, ya que la potencia fue menor del $60 \%$ y, la no aleatorización de los grupos, propios de un diseño cuasiexperimental, que no utiliza ningún tipo de cegamiento; siendo necesario realizar réplicas del estudio cumpliendo con los requisitos de los estudios randomizados y de gran porte. Mientras tanto, constituye una muestra de lo que sucede en el contexto hospitalario local en la atención del neonato y que sí sería posible su aplicación rutinaria, si se ofrecen todas las condiciones apropiadas: infraestructura, materiales y recurso humano capacitado, mejorando las condiciones de los establecimientos de salud del primer nivel de atención, sobre todo por el déficit de recurso humano que padecen estos centros y que puede constituirse en justificativa para los profesionales.

\section{Declaración de financiamiento y de conflictos de interés:}

El estudio fue financiado por los autores; declaran no tener conflictos de interés.

\section{Contribución de autoría:}

CMRM, MPLC: Concepción y diseño del estudio, recolección de los datos, análisis e interpretación de los resultados y aprobación de la versión que será publicada. 


\section{REFERENCIAS BIBLIOGRÁFICAS}

1. Gangal P, Bhagat K, Prabhu S, Nair R. Breast crawl initiation of breastfeeding by breast crawl [internet]. Mumbai-India: UNICEF Maharashtra; 2007 [citado 03 de mar 2010]. 41p. Disponible en: http://www.breastcrawl.org/pdf/breastcrawl.pdf

2. Edmond KM, Zandoh C, Quigley MA, Amenga-Etego S, Owusu-Agyei S, Kirkwood BR. Delayed breastfeeding initiation increases risk of neonatal mortality. Pediatrics [serie de internet]. Mar 2006 [citado mar 15 de 2011]; 117(3):380-86. Disponible en: http://pediatrics.aappublications.org/content/pe diatrics/117/3/e380.full.pdf

3. Pinto LF. Apego y Lactancia natural. Rev. Chil Pediatría [serie de internet]. 2007 [citado 29 de ene 2010]. 78 (Supl.1):96-102. Disponible en: http://www.scielo.cl/pdf/rcp/v78s1/art08.pdf

4. Moore E, Anderson GC, Bergman N, Donwswills T. Early skin-to-skin contact for mothers and their healthy newborn infants [internet]. Britain: Cochrane Library. Jul 2012 [citado 24 de sep 2013]. Disponible en: http://onlinelibrary.wiley.com/doi/10.1002/146 $\underline{\text { 51858.CD003519.pub2/full }}$

5. Chaturvedi P. Breast crawl to initiate breast feeding whiting half an hour after birth. J MGINS [serie de internet]. Sep 2008 [citado 10 de mar 2012]; 13(ii):9-14. Disponible en: http://medind.nic.in/jaw/t08/i2/jawt08i2p9.pdf

6. Organización Panamericana de la Salud (OPS). Más allá de la supervivencia: Prácticas integrales durante la atención del parto, beneficiosas para la nutrición y la salud de madres y niños [internet]. Washington: OPS; 2007 [actualizado s.f.; citado 20 de jun 2010]. Disponible en: http://www1.paho.org/hq/dmdocuments/2009/ Mas-alla-de-la-supervivencia-Practicasatencion-parto.pdf?ua=1

7. Gispert C. Nuevo Manual de la Enfermería. España Científico. 2009. p.762.

8. Conde-Agudelo A, Diaz-Rosello JL. Kanguroo mother care to reduce morbidity an mortality in low birthweight infants. [citado 15 de may 2015]. In: Cochrane Database System Rev [internet]. Britain: Cochrane Library. Apr 2014
(4): CD002771. Disponible en: http://onlinelibrary.wiley.com/doi/10.1002/146 51858.CD002771.pub3/epdf

9. Alba RC, Aler GI, Olza FI. Maternidad y salud: ciencia, consciencia y experiencia [internet]. Madrid: Ministerios de Sanidad, Servicios Sociales e Igualdad; 2012 [citado 10 de mar 2013]. 168p. Disponible en: http://www.msssi.gob.es/organizacion/sns/plan CalidadSNS/pdf/maternidadSaludCiencia.pdf

10. Ministerio de Salud. Atención Integral de la Salud Neonatal. Norma Técnica [internet]. Lima: MINSA; 2015 [citado 23 mar 2015]. 31p. Disponible: http://bvs.minsa.gob.pe/local/minsa/3281.pdf

11. Crenshaw J, Care practice \#6. No separation of mother and baby, with unlimited opportunities for breastfeeding. J Perinat Educ [serie de internet]. May 2007 [citado may de 2014], 16(3):39-43. Disponible en: https://www.ncbi.nlm.nih.gov/pmc/articles/PM $\underline{\mathrm{C} 1948089 /}$

12. Klaus M. Mother and infant: early emotional ties. Pediatrics [serie de internet]. Nov 1998 [citado mar de 20 2011]; 102(5):1244-46. Disponible en: http://www.breastcrawl.org/pdf/mother-and$\underline{\text { Infant-early-emotional-ties.pdf }}$

13. Bergstrom A, Okong P, Ransjo-Aedvison AB. Inmediate maternal thermal response to skinto-skin care of newborn. Acta Paediatrica [serie de internet]. 2007 [citado 15 de may 2014]; 96: 655-8. Disponible en: https://www.researchgate.net/publication/6367 310_Immediate_maternal_thermal_response_to _skin-to-skin_care of newborn

14. Rivara D G, Rivara D P, Cabrejos K, Quiñones M, Ruiz S, Miñano K, et al. Contacto piel a piel inmediato: efecto sobre el estado de ansiedad y depresión materna posparto y sobre la adaptabilidad neonatal hacia la lactancia materna precoz. Rev. peru. pediatr [serie de internet]. 2007 [citado 18 de ene 2010]; 60(3):140-149. Disponible en: http://sisbib.unmsm.edu.pe/BVRevistas/rpp/v6 0n3/pdf/a02v60n3.pdf 
15. Lawn JE, Cousens S, Zupan J. 4 million neonatal deaths: When? Where? Why? The Lancet [serie de internet]. Mar 2005 [citado: 15 abr 2011]; 365(9462): [aproximadamente 10 laudas]. Disponible en: http://www.mamaye.org/sites/default/files/evid ence/lancet_neonatal_survival_paper1.pdf

16. Ayukarningsih Y, Dwinanda A. Early initiation of breasthfeeding a Dustira Hospital. Paediatr Indones [serie de internet]. May 2015 [citado 20 de may 2015]; 55(3):126-130. Disponible en:

https://doaj.org/article/fd56b492022f4f7dbe7e6 b87ed1a541a

17. Guzmán VFP. Calidad del contacto precoz entre la madre y su recién nacido a término vigoroso atendidos en el Instituto Materno Perinatal en el mes de mayo 2015 [tesis de licenciatura]. Lima-Perú: Universidad Nacional Mayor de San Marcos. 55p.

18. UNICEF. La leche materna salva vidas. El Universal [internet]. Méxco: DONAUNICEF; abr de 252015 [citado 15 may 2015]. 08p. Disponible en: https://www.unicef.org/mexico/spanish/UNICE F_SuplementoAbril2015.pdf 